\title{
EDITORIAL
}

\section{State of the Science in SCI Rehabilitation}

Y Chen

University of Alabama at Birmingham,

Birmingham, AL, USA

E-mail: yychen@uab.edu

Dear Spinal Cord Reader,

This issue of the Spinal Cord is entirely dedicated to the presentations and discussions from the State of Science Conference in Spinal Cord Injury Rehabilitation held in June 2011 in Washington DC, USA, in conjunction with the joint annual meetings of the International Spinal Cord Society and the American Spinal Injury Association. If you were there, I am sure you would agree that the conference was unprecedented, full of energy and global passions for better sciences and clinical care of persons with spinal cord injury (SCI). If you unfortunately missed it, a set of 10 articles published in this issue will do the trick.

The Introduction by the Steering Committee of the State of the Science Conference illustrates the purposes and program content of the conference as well as the behind-the-scene information. A total of eight articles, two each, are prepared for the four conference themes. For Neurologic and Functional Recovery, Galea's article provides an evidence review of the effect of various rehabilitation approaches on health improvement and maintenance after SCI, including fitness, circulation, body composition, nervous system and physical functioning. In Curt's report, a number of tangible targets were proposed for translational dialogs between scientists and clinicians and also for future SCI research.

For technology for mobility and function, Boninger et al. examine the current and future technology advances and discuss their potential impact on SCI, using the conceptual framework of the International Classification of Function. The commentary by Humayun and Weiland affirms and extends Boninger's views on future technology development, barriers and associated socioeconomic challenges.

DeVivo's article addresses aging and secondary conditions by reviewing the global epidemiology of SCI and projecting the future trends in the incidence, prevalence, demographic and injury profile, as well as selected outcomes. Jensen et al propose a working definition and conceptual model for secondary health conditions in attempting to standardize reporting of aging research and improve the evidence base and knowledge translation.

For Psychosocial, Vocational and Quality of Life Outcomes, Frieden and Winnegar provide multidimensional overview of vocational issues and strategies to improve employment after SCI, including flexible work environments, selection of jobs that appropriately meet personal physical capacity, knowledge of job market, education and skill training. Post and van Leeuwen report a systemic review of subject well-being after SCI, associated psychosocial predictors and unmet needs for research.

Heinemann et al. concluded this issue with recommendations that define SCI rehabilitation research over the next decade, based on the feedback from keynote and plenary presentations, panel and small group discussions, and input through a website. A White House meeting, sponsored by the National Institute on Disability and Rehabilitation Research, US Department of Education and the Interagency Committee on Disability Research, was held in November, 2011 for interested stakeholders to review and discuss these recommendations. We hope that these recommendations will be adopted by countries and regions around the world and stimulate national and international cross-disciplinary collaboration in SCI research. We look forward to seeing the fruits of these efforts in improved care and outcomes of the population we serve.

Spinal Cord (2012) 50, 341; doi:10.1038/sc.2012.44 